\title{
ADDRESSING THE CHALLENGES OF IMPLEMENTATION OF HIGH-ORDER FINITE-VOLUME SCHEMES FOR ATMOSPHERIC DYNAMICS ON UNSTRUCTURED MESHES
}

\author{
Panagiotis Tsoutsanis $^{1}$, Dimitris Drikakis ${ }^{2}$ \\ ${ }^{1}$ Fluid Mechanics and Computational Sciences,Cranfield University \\ Cranfield MK43 0AL, United Kingdom \\ e-mail: panagiotis.tsoutsanis@cranfield.ac.uk \\ ${ }^{2}$ University of Strathclyde \\ Glasgow G1 1XW, United Kingdom \\ e-mail: dimitris.drikakis@strath.ac.uk
}

Keywords: WENO, Unstructured Meshes, Non-Hydrostatic, Rising Bubble

\begin{abstract}
The solution of the non-hydrostatic compressible Euler equations using Weighted Essentially Non-Oscillatory (WENO) schemes in two and three-dimensional unstructured meshes, is presented. Their key characteristics are their simplicity; accuracy; robustness; non-oscillatory properties; versatility in handling any type of grid topology; computational and parallel effciency. Their defining characteristic is a non-linear combination of a series of high-order reconstruction polynomials arising from a series of reconstruction stencils. In the present study an explicit TVD Runge-Kutta $3^{\text {rd }}$-order method is employed due to its lower computational resources requirement compared to implicit type time advancement methods. The WENO schemes (up to $5^{\text {th }}$-order) are applied to the two dimensional and three dimensional test cases: a $2 D$ rising thermal bubble. The scalability and efficiency of the schemes is also investigated.
\end{abstract}




\section{Introduction}

Understanding the atmospheric processes both qualitatively and quantitatively on regional and global scales is of great value for scientists and policy decision makers. Tools utilised by the scientific community to gain insight into the complex atmosphere-climate system, include the numerical weather prediction (NWP) and climate models. The main objective is to accurately simulate weather and climate variations, trends, patterns etc. All the models utilised for this purpose have a distinctive component in common, the dynamical core, which solves the fluid dynamics equations. In other words, it is the model component that drives atmospheric motions, hence with a profound impact on the accuracy of the predictions. The leap occurring in computing processing power the last decade, provides a motivation to resolve atmospheric processes on finer scales and higher resolution than happened before. There is currently a trend of either enhancing the majority of the dynamical cores of established models or develop new dynamical cores that utilise highly sophisticated algorithms or set of equations that are tailored for finer resolution simulations in larger computing facilities. A number of initiatives have been taken to address this bottleneck and develop the next generation of dynamical cores. Such initiatives include the UK Met Office and European Centre for Medium-Range Weather Forecasts Even Newer Dynamics for General Atmospheric Modelling of the Environment (ENDGame) [51]; the National Center for Atmospheric Research High-Order Methods Modelling Environment [34]; the National Oceanic and Atmospheric Administration AM3 model [35] and the Nonhydrostatic Icosahedral Model (NIM) [36]; the Frontier Research Center for Global Change Nonhydrostatic Icosahedral Atmospheric Model, (NICAM) [41]; the Non-hydrostatic Unified Model of the Atmosphere (NUMA) [17]; and the Max Planck Institute for Meteorology and Deutscher Wetterdienst ICOsahedral Non-hydrostatic general circulation models, (ICON) [31].

This paper focuses on the the numerics of the dynamical core aspect of atmospheric models employed for weather and climate predictions for well established test cases. Since high performance computing systems enable the analysis on finer scales and higher resolution, the topography should be efficiently and accurately represented, a property typical for unstructured grids. In the past, finite volume numerical methods have been introduced in the structured grid context resulting in robust schemes that enjoyed a wide recognition in various disciplines, including the weather and climate prediction community, e.g. [27, 28, 45].

The first generation numerical methods for unstructured grids exhibited lower accuracy and were computationally more demanding than structured grids. However, the numerical methods for unstructured grids have matured and numerous elegant approaches $[10,11,18,21,29,37$, 39, 47, 49, 50, 56-58] and algorithms have been developed in the finite volume framework for a wide range of applications for Computational Fluid Dynamics(CFD). Other state-of-theart approaches are also available, such as the Discontinuous Galerkin (DG) [6, 7, 11, 52, 57, 64], and Spectral Finite-Volume (SFV) methods [5, 53-55, 58, 63] that have been successfully applied for CFD applications. For the finite volume framework the first class of high-resolution methods developed for unstructured grids included the ENO type schemes [1, 46], followed by the WENO type schemes [13, 23, 42, 43]. In the WENO case, the high-order accuracy was achieved by non-linearly combining a series of high-order reconstruction polynomials arising from a series of reconstruction stencils. Recently, a class of WENO type methods [47, 49] 
has been successfully extended to hybrid unstructured meshes with various geometrical shapes such as tetrahedrals, hexahedrals, prisms, and pyramids. The schemes can achieve the very high order of spatial accuracy across interfaces between cells of different types, and the same time essentially non-oscillatory profiles are produced for discontinuous solutions. This gives greater flexibility to handle complex geometrical shapes in an efficient and accurate manner.

For the atmospheric dynamics there is also an overwhelming number of recent state-ofthe-art approaches that utilise high-order schemes for either structured grid or unstructured dynamical cores $[4,7,14,15,24,30,32,44,59-61]$. Regarding the high-order schemes (higher than 3rd order of spatial accuracy) for unstructured dynamical cores all of the approaches are based on the Discontinuous Galerkin framework and the Lagrange-Galerkin Spectral Element Method[7, 15, 26, 32, 60]. The fact that WENO schemes have been successfully applied to a range of smooth and discontinuous flows [10, 11, 50], including the Reynolds Averaged Navier Stokes (RANS) equations [2, 3, 8], motivates the application of these schemes in the context of non-hydrostatic Euler equations.

The aim of this paper is to assess the performance and suitability of WENO-class higherorder finite volume schemes in conjunction with unstructured meshes for well established test cases for non-hydrostatic Euler equations. The schemes can be used in any type of conforming grids.

The paper is organised as follows. In Section 2 the governing equations of a dry nonhydrostatic atmosphere are presented. Section 3 outlines the spatial discretisation techniques WENO reconstruction, numerical fluxes, source terms and temporal discretisation. The results obtained with various schemes for different test cases, including discussion on the parallel performance, are presented in Section 4 . Section 5 summarises the conclusions drawn from the present study.

\section{Governing Equations}

The compressible inviscid Euler equations employed by the CFD community where mass, momentum and total energy are the conserved quantities, are rarely used for atmospheric studies, with a few notable exceptions [16]. The primary reason for not being that common in atmospheric studies is the additional computational step required to compute potential temperature from total energy. However, the benefits of using these set of equations are their formally conservative nature as reported in [16]. The compressible non-hydrostatic Euler equations without moisture effects are considered in the following form:

$$
\frac{\partial}{\partial t} \mathbf{U}+\frac{\partial}{\partial x} \mathbf{F}(\mathbf{U})+\frac{\partial}{\partial y} \mathbf{G}(\mathbf{U})+\frac{\partial}{\partial z} \mathbf{H}(\mathbf{U})=\mathbf{S},
$$

where $\mathbf{U}$ is the vector of the conserved variables, $\mathbf{F}, \mathbf{G}, \mathbf{H}$ are the flux vectors in $x, y$ and $z$ directions of Cartesian coordinates and $\mathbf{S}$ is a gravity source function given by 


$$
\begin{gathered}
\mathbf{U}=\left[\begin{array}{c}
\rho \\
\rho u \\
\rho v \\
\rho w \\
E
\end{array}\right], \quad \mathbf{F}=\left[\begin{array}{c}
\rho u \\
\rho u^{2}+p \\
\rho u v \\
\rho u w \\
u(E+p)
\end{array}\right] \\
\mathbf{H}=\left[\begin{array}{c}
\rho w \\
\rho w u \\
\rho w v \\
\rho w^{2}+p \\
w(E+p)
\end{array}\right], \quad \mathbf{G}=\left[\begin{array}{c}
\rho v \\
\rho v u \\
\rho v^{2}+p \\
\rho v w \\
v(E+p)
\end{array}\right] . \\
\mathbf{S}=\left[\begin{array}{c} 
\\
0 \\
0 \\
0 \\
-\rho g \\
0
\end{array}\right] .
\end{gathered}
$$

where $\rho$ is density; $u, v, w$ are the velocity components in the $x, y$ and $z$ directions of Cartesian coordinates, respectively; $p$ is pressure; $E=\rho c_{v} T+(1 / 2) \rho\left(u^{2}+v^{2}+w^{2}\right)+\rho g y$ is the total energy per unit mass; $\gamma$ is the ratio of specific heats; $g$ the gravitational constant; $g y$ is the geopotential height; $T$ is the temperature; $R=c_{p}-c_{v}$ is the gas constant, where $c_{p}$ is the specific heat at constant pressure and $c_{v}$ is the specific heat at constant volume. The ideal gas law is used with $\gamma=1.4$ throughout this work, with the pressure $p$ given by

$$
p=p_{s}\left(\frac{\rho R \theta}{p_{s}}\right)^{\gamma},
$$

where $p_{s}$ is the atmospheric pressure at sea level, and $\theta$ is the potential temperature

$$
\theta=\frac{T}{\pi}
$$

with $\pi$ being the Exner pressure provided by the following expression

$$
\pi=\left(\frac{p}{p_{s}}\right)^{\frac{R}{c_{p}}} .
$$

The following equations relate the Exner pressure $\pi$ to the density $\rho$, and the potential temperature $\theta$ to the total energy per unit mass $E$ :

$$
\rho=\frac{p_{s}}{R \theta} \pi^{\frac{c_{v}}{R}}
$$

and

$$
E=\rho c_{v} \theta \pi+(1 / 2) \rho\left(u^{2}+v^{2}+w^{2}\right)+\rho g y
$$


The Exner pressure and potential temperature can be split in their mean and perturbed parts as follows

$$
\theta(x, y, z, t)=\bar{\theta}(y, t)+\theta^{\prime}(x, y, z, t)
$$

and

$$
\pi(x, y, z, t)=\bar{\pi}(y, t)+\pi^{\prime}(x, y, z, t),
$$

where the hydrostatic balance of their mean values is given by

$$
c_{p} \bar{\theta} \frac{d \bar{\pi}}{d y}=-g
$$

Integrating in space over a mesh element $V_{i}$, the following semi-discrete finite-volume method is obtained

$$
\frac{d}{d t} \mathbf{U}_{i}+\frac{1}{\left|V_{i}\right|} \oint_{\partial V_{i}} \mathbf{F}_{n} d A=\mathbf{S}_{\mathbf{i}}
$$

and using the rotational invariance property of the Euler equations [48]

$$
\mathbf{F}_{n}(\mathbf{U})=\mathbf{F}(\mathbf{U}) n_{x}+\mathbf{G}(\mathbf{U}) n_{y}+\mathbf{H}(\mathbf{U}) n_{z}=\mathbf{T}^{-\mathbf{1}} \mathbf{F}(\mathbf{T} \mathbf{U}),
$$

where $\mathbf{n}=\left(n_{x}, n_{y}, n_{z}\right)$ is the outward unit normal vector; $\mathbf{U}_{i}(t)$ are the cell averages of the solution at time $t ; \mathbf{F}_{n}$ is the projection of the flux tensor on the normal direction; $\mathbf{T}$ is the rotation matrix; and $\mathbf{T}^{-1}$ its inverse [48] ; and the source term $\mathbf{S}_{\mathbf{i}}$ is given by

$$
\mathbf{S}_{\mathbf{i}}=\frac{1}{\left|V_{i}\right|} \int_{V_{i}} S(x, y, z, t) d x d y d z
$$

Assuming that the element's surface consists of $L$ faces, with the spatial index $i$ being omitted for simplicity,

$$
\partial V_{i}=\sum_{j}^{L} A_{j}
$$

( $\mathbf{n}_{j}$ denoting the outward unit vector for face $A_{j}$ ), then the integral over the element boundary $\partial V_{i}$ can be split into the sum of integrals over each face, resulting in the following expression:

$$
\frac{d}{d t} \mathbf{U}_{i}=\mathbf{R}_{i}
$$

where

$$
\mathbf{R}_{i}=-\frac{1}{\left|V_{i}\right|} \sum_{j=1}^{L} \int_{A_{j}} \mathbf{F}_{n, j} d A+\mathbf{S}_{\mathbf{i}}=-\frac{1}{\left|V_{i}\right|} \sum_{j=1}^{L} \mathbf{K}_{i j}+\mathbf{S}_{\mathbf{i}} .
$$

The numerical flux $\mathbf{K}_{i j}$ corresponding to face $j$ of the cell $V_{i}$ is the surface integral of the projection of the tensor of fluxes onto $\mathbf{n}_{j}$. In a numerical method the exact integral expression for the numerical flux $\mathbf{K}_{i j}$ for the face $j$ of a cell $V_{i}$ is approximated by a suitable Gaussian 
numerical quadrature where the wall bounded elements quadrature points are depicted with different color corresponding to different weights

$$
\mathbf{K}_{i j}=\int_{A_{j}} \mathbf{F}_{n, j} d A=\sum_{\beta} \mathbf{F}_{n, j}\left(\mathbf{U}\left(\mathbf{x}_{\beta}, t\right)\right) \omega_{\beta}\left|A_{j}\right|,
$$

where the subscript $\beta$ corresponds to different Gaussian integration points $\mathbf{x}_{\beta}$ and weights $\omega_{\beta}$ over the face $A_{j}$.

\section{Numerical Framework}

In this section the numerical framework for the solution of the compressible non-hydrostatic Euler equations is presented with respect to spatial and temporal discretisation.

\subsection{Spatial discretisation}

The spatial discretisation is based on the approach of [47, 49], which is suitable for unstructured meshes with various types of element shapes in 2D and 3D, and it has been previously used successfully for laminar, transitional and turbulent flows [50]. Therefore, only the key characteristics of this approach are going to be described in this paper. The first step in the approximation of the governing equations concerns the spatial discretisation of the domain $\Omega \subset R^{3}$ into $E$ number of conforming elements $V_{i}$, with the index $i$ ranging from 1 to $E$ number of elements. The elements can be any combination of hexahedrals, pyramids, prisms, and tetrahedrals as shown in Figure 1 in 3D, and triangles or quadrilaterals in 2D. It must be noted that the present WENO schemes are not limited by conforming meshes since they can be used with non-conforming meshes which brings additional benefits such as adaptive mesh refinement [12].

The combination of all elements in the spatial domain is given by

$$
\Omega=\bigcup_{i=1}^{E} V_{i}
$$

In the context of the finite volume framework that is employed in the present study, the data is represented by cell averages of conserved variable $u(x, y, z)$ in each element $V_{i}$

$$
\bar{u}_{i}=\frac{1}{\left|V_{i}\right|} \int_{V_{i}} u(x, y, z) d V,
$$

where $\left|V_{i}\right|$ is the volume of the element. To achieve high-order, accurate spatial discretisation, high-order accurate point-wise values of the solution need to be recovered from the cell averages. The reconstruction problem can thus be reformulated as follows: For a target cell $V_{0}$ we build a high-order polynomial $p_{i}(x, y, z)$ that has the same cell average as $u$ on the target cell

$$
\bar{u}_{i}=\frac{1}{\left|V_{i}\right|} \int_{V_{i}} u(x, y, z) d V=\frac{1}{\left|V_{i}\right|} \int_{V_{i}} p_{i}(x, y, z) d V .
$$


The point-wise values of the conserved variable $u$ in each cell are approximated by a polynomial of a desired order of accuracy $r$. The polynomial uses the cell averages of $u(x, y, z)$ on the target cell $V_{i}$ as well as averages $\bar{u}_{m}$ from neighbouring cells, $V_{m}$. The key

\subsubsection{Reconstruction algorithm}

The main steps for the reconstruction process are the following:

1. For all the non tetrahedral cells the cell is decomposed into tetrahedral elements. It must be stressed that the decomposition to tetrahedral cells, is done only for the transformation purposes to the reference space in order to remove any scaling effect of the least square system to be solved.

2. Choose one of the resulting decomposed elements.

3. Transform the chosen decomposed element from the physical space described by the Cartesian coordinates $x, y, z$ into a reference space described by $\xi, \eta, \zeta$.

4. Based on the Jacobian matrix of the transformation of the chosen decomposed element, map the coordinates of the entire element into the reference space described by coordinates $\xi, \eta, \zeta$.

5. Based on the same Jacobian, all the elements in the stencil are transformed to the reference space and their volumes, and barycentres positions are recomputed in the new reference space.

Let $\mathbf{v}_{i j}, j=1,2, \ldots J_{i}$ be the vertices of the considered (general) element, which can be either tetrahedral, hexahedral, prismatic or pyramidal. Let also $\mathbf{w}_{1}=\left(x_{1}, y_{1}, z_{1}\right), \mathbf{w}_{2}=\left(x_{2}, y_{2}, z_{2}\right)$, $\mathbf{w}_{3}=\left(x_{3}, y_{3}, z_{3}\right), \mathbf{w}_{4}=\left(x_{4}, y_{4}, z_{4}\right)$ be the four vertices of one of the tetrahedrals of this element. Obviously, these vertices are between the $\mathbf{v}_{i j}$ ones. The transformation from the Cartesian coordinates $x, y, z$ into a reference space $\xi, \eta, \zeta$ is given by the following equations

$$
\left(\begin{array}{l}
x \\
y \\
z
\end{array}\right)=\left(\begin{array}{l}
x_{1} \\
y_{1} \\
z_{1}
\end{array}\right)+J \cdot\left(\begin{array}{l}
\xi \\
\eta \\
\zeta
\end{array}\right),
$$

with the Jacobian matrix given by

$$
J=\left[\begin{array}{ccc}
x_{2}-x_{1} & x_{3}-x_{1} & x_{4}-x_{1} \\
y_{2}-y_{1} & y_{3}-y_{1} & y_{4}-y_{1} \\
z_{2}-z_{1} & z_{3}-z_{1} & z_{4}-z_{1}
\end{array}\right] .
$$

Using an inverse mapping the element $V_{i}$ can be transformed to the element $V_{i}^{\prime}$ in the reference co-ordinate system

$$
\mathbf{v}_{i j}^{\prime}=J^{-1} \cdot\left(\mathbf{v}_{i j}-\mathbf{w}_{1}\right), \quad j=1,2, \ldots J_{i} .
$$


The spatial average of $u(x, y, z)$ does not change during transformation

$$
\bar{u}_{i}=\frac{1}{\left|V_{i}\right|} \int_{V_{i}} u(x, y, z) d V \equiv \frac{1}{\left|V_{i}^{\prime}\right|} \int_{V_{i}^{\prime}} u(\xi, \eta, \zeta) d \xi d \eta d \zeta .
$$

For performing the reconstruction on the target element $V_{i}$, we form the central reconstruction stencil $\mathcal{S}$ as described previously, consisting of $M+1$ elements, including the target element $V_{i}$

$$
\mathcal{S}=\bigcup_{m=0}^{M} V_{m}
$$

where the index $m$ refers to the local numbering of the elements in the stencil, with the element with index 0 being the considered cell $i$. The $r^{\text {th }}$ order reconstruction polynomial at the transformed cell $V_{0}^{\prime}$ is sought as an expansion over local polynomial basis functions $\phi_{k}(\xi, \eta, \zeta)$

$$
p(\xi, \eta, \zeta)=\sum_{k=0}^{K} a_{k} \phi_{k}(\xi, \eta, \zeta)=\bar{u}_{0}+\sum_{k=1}^{K} a_{k} \phi_{k}(\xi, \eta, \zeta),
$$

where $a_{k}$ are degrees of freedom and the upper index in the summation of expansion $K$ is related to the order of the polynomial $r$ by the expression $K=\frac{1}{6}(r+1)(r+2)(r+3)-1$ for $3 \mathrm{D}$ and $K=\frac{1}{2}(r+1)(r+2)-1$ for 2D. The conservation condition (16) imposes an important constraint on the basis functions: they must have zero mean value over the cell $V_{0}^{\prime}$. On purely tetrahedral meshes hierarchical orthogonal reconstruction basis functions [10, 57], defined on the reference element, satisfy this requirement automatically. Since our general cells are not necessarily transformed onto a unit tetrahedron or cube, we need to construct basis functions $\phi_{k}$ in such a way that the condition is satisfied identically, irrespective of the degrees of freedom. The basis functions are defined as follows:

$$
\phi_{k}(\xi, \eta, \zeta) \equiv \psi_{k}(\xi, \eta, \zeta)-\frac{1}{\left|V_{0}^{\prime}\right|} \int_{V_{0}^{\prime}} \psi_{k} d \xi d \eta d \zeta, \quad k=1,2, \ldots
$$

Any type of orthogonal polynomial basis function can be utilised; however, in the present study generic polynomial basis functions are used

$$
\left\{\psi_{k}\right\}=\xi, \eta, \zeta, \xi^{2}, \eta^{2}, \zeta^{2}, \xi \cdot \eta, \xi \cdot \zeta, \zeta \cdot \eta, \xi \cdot \eta \cdot \zeta \ldots
$$

To find the unknown degrees of freedom $a_{k}$ for each cell $V_{m}^{\prime}$ from the stencil the cell average of the reconstruction polynomial $p(\xi, \eta, \zeta)$ must be equal to the cell average of the solution $\bar{u}_{m}$ :

$$
\int_{E_{m}^{\prime}} p(\xi, \eta, \zeta) d \xi d \eta d \zeta=\left|V_{m}^{\prime}\right| \bar{u}_{0}+\sum_{k=1}^{K} \int_{V_{m}^{\prime}} a_{k} \phi_{k} d \xi d \eta d \zeta=\left|V_{m}^{\prime}\right| u_{m}, \quad m=1, \ldots
$$

Denoting the integrals of the basis function $k$ over the cell $m$ in the stencil, the vector of right-hand side by $A_{m k}$ and $\mathbf{b}$ are given by 


$$
A_{m k}=\int_{V_{m}^{\prime}} \phi_{k} d \xi d \eta d \zeta, \quad b_{m}=\left|V_{m}^{\prime}\right|\left(\bar{u}_{m}-\bar{u}_{0}\right)
$$

We can rewrite the equations for degrees of freedom $a_{k}$ in a matrix form as

$$
\sum_{k=1}^{K} A_{m k} a_{k}=b_{m}, \quad m=1,2, \ldots M
$$

The three-dimensional integrals on the left-hand side of (26) are calculated using Gaussian quadratures of appropriate order. In general, to compute the degrees of freedom $a_{k}$ we need at least $K$ cells in the stencil, different from the target cell $E_{0}$. However, the use of the minimum possible number of cells in the stencil $M \equiv K$ results in a scheme which may become unstable on general meshes [9, 21, 22, 49]. It is, therefore, recommended to use more cells in the stencil than the minimum required number. Although it is usually sufficient to use $50 \%$ more cells, for mixed-element meshes it is safer to increase the stencil further. We typically select $M=2 \cdot K$.

Since the resulting system becomes over-determined, the least-square procedure is invoked to solve it. The least-square reconstruction is obtained by seeking the minimum of the following functional

$$
\mathcal{F}=\sum_{m=1}^{M} w_{m} \cdot\left(\sum_{k=1}^{K} A_{m k} a_{k}-b_{m}\right)^{2}
$$

where the weights $w_{m}$ are squared reciprocals of the distance between cells $E_{0}^{\prime}$ and $E_{m}$. The advantage of the weighted least square reconstruction is that the influence of the data further from the considered $E_{0}^{\prime}$ is reduced, although a central least square reconstruction is materialised with the weights $\omega_{m}$ being equal to unity. Minimisation of $\mathcal{F}$ gives a linear system for finding $a_{k}$ :

$$
\sum_{k=1}^{K} C_{k} a_{k}=\sum_{m=1}^{M} A_{m p} w_{m} b_{m}, \quad C_{k}=\left(\sum_{m=1}^{M} w_{m} A_{m k} A_{m p}\right), \quad p=1, \ldots K
$$

A QR decomposition method is employed to solve this system of equations. The coefficients of the resulting linear symmetric matrix A are pre-computed and stored for each element during the pre-processing stage of the calculation, thus increasing the computational efficiency of the method. If the mesh was to be refined these would automatically require the need to recompute and store this matrix. Having solved numerically the linear system, we can form the reconstruction polynomial (23).

\subsubsection{WENO reconstruction}

The main characteristic of the WENO reconstructions is the use of reconstruction polynomials from several different stencils and their combination in a non-linear manner. In WENO schemes the actual reconstructed value is a convex combination of reconstructed values from 
stencils, with nonlinear (solution-adaptive) WENO weights [23, 42, 62]. These nonlinear weights are constructed from the linear (constant) weights by taking into account the smoothness of the solution in each of the reconstruction stencils. The resulting methods are uniformly high-order accurate, while maintaining non-oscillatory behaviour in regions with sharp gradients. The WENO reconstruction used in the present study is based on the implementation of [49], where the reader is referred to for further details.

\subsubsection{Numerical fluxes and Source term}

Since for each computational cell the point-wise values of the conserved vector $\mathbf{U}$ are replaced by high-order reconstruction polynomials the numerical flux for the face $A_{j}$ of cell $V_{i}$ is discontinuous at each Gaussian point $\beta$. The values $\mathbf{U}_{\beta}^{-}$and $\mathbf{U}_{\beta}^{+}$correspond to the reconstructed value of cell $V_{i}$ and its adjacent neighbour $V_{i^{\prime}}$. In upwind finite-volume methods the discontinuity is replaced at each Gaussian integration point by using a monotone function of left and right boundary extrapolated values so that (14) can be re-written as

$$
\mathbf{K}_{i j} \approx \sum_{\beta} \mathbf{F}_{n, j}\left(\mathbf{U}_{\beta}^{-}, \mathbf{U}_{\beta}^{+}\right) \omega_{\beta}\left|A_{j}\right| .
$$

The function $\tilde{\mathbf{F}}_{n, j}\left(\mathbf{U}_{\beta}^{-}, \mathbf{U}_{\beta}^{+}\right)$is the Riemann solver [48]. Employing the rotational invariance property of the Euler equations [48], for each face $A_{j}$ the normal projection of the flux tensor $\mathbf{F}_{n, j}$ is replaced by

$$
\mathbf{F}_{n, j}=\mathbf{T}^{-1} \mathbf{F}\left(\mathbf{T}_{j} \mathbf{U}\right),
$$

where $\mathbf{T}_{j}$ is the rotation matrix for face $j$. Re-writing (28) for $\mathbf{K}_{i j}$ gives

$$
\mathbf{K}_{i j}=\sum_{\beta} \mathbf{F}_{n, j}\left(\mathbf{U}_{\beta}^{-}, \mathbf{U}_{\beta}^{+}\right) \omega_{\beta}\left|A_{j}\right|=\sum_{\beta} \mathbf{T}^{-\mathbf{1}} \mathbf{F}\left(\hat{\mathbf{U}}_{L}, \hat{\mathbf{U}}_{R}\right) \omega_{\beta}\left|A_{j}\right|,
$$

where $\hat{\mathbf{U}}_{j}$ is the rotated conserved variable and

$$
\hat{\mathbf{U}}_{L}=\mathbf{T}_{j} \mathbf{U}_{\beta}^{-}, \quad \hat{\mathbf{U}}_{R}=\mathbf{T}_{j} \mathbf{U}_{\beta}^{+} .
$$

The flux function for the Gaussian point $\beta$ results in the one-dimensional Riemann initial value problem

$$
\frac{\partial}{\partial t} \hat{\mathbf{U}}+\frac{\partial}{\partial s} \hat{\mathbf{F}}=\mathbf{0}, \quad \hat{\mathbf{F}}=\mathbf{F}(\hat{\mathbf{U}}), \quad \hat{\mathbf{U}}(s, 0)=\begin{gathered}
\hat{\mathbf{U}}_{L}, \quad s<0, \\
\hat{\mathbf{U}}_{R}, \quad s>0
\end{gathered}
$$

In the present study the HLLC Riemann solver of Toro [48] is employed. The numerical source term $\mathrm{S}_{\mathbf{i}}$ is approximated by using a Gaussian quadrature rule of the same order of accuracy as the order of the polynomial of the reconstruction

$$
\mathbf{S}_{\mathbf{i}} \approx \frac{1}{\left|V_{i}\right|} \sum_{\beta} \mathbf{S}\left(x_{\beta}, y_{\beta}, z_{\beta}, t\right) w_{\beta},
$$

where $x_{\beta}, y_{\beta}, z_{\beta}$ is the volumetric Gaussian quadrature points and $w_{\beta}$ is the Gaussian weights of $r$-order of accuracy. 


\subsection{Temporal discretisation}

Having constructed the numerical fluxes $\mathbf{F}_{n, j}$ as expressed in the semi-discrete conservative formulation, the next step involves the advancement of the solution in time. The explicit SSP Runge-Kutta $3^{\text {rd }}$-order method [19] has been employed for the time integration

$$
\begin{gathered}
\mathbf{U}_{\mathbf{i}}^{1}=\mathbf{U}_{\mathbf{i}}{ }^{n}+\Delta t \cdot \mathbf{R}_{i}\left(\mathbf{U}_{\mathbf{i}}{ }^{n}\right) \\
\mathbf{U}_{\mathbf{i}}{ }^{2}=\frac{3}{4} \mathbf{U}_{\mathbf{i}}{ }^{n}+\frac{1}{4} \mathbf{U}_{\mathbf{i}}{ }^{1}+\frac{\Delta t}{4} \cdot \mathbf{R}_{i}\left(\mathbf{U}^{1}\right) \\
\mathbf{U}_{\mathbf{i}}{ }^{n+1}=\frac{1}{3} \mathbf{U}_{\mathbf{i}}{ }^{n}+\frac{2}{3} \mathbf{U}_{\mathbf{i}}{ }^{2}+\frac{2 \Delta t}{3} \cdot \mathbf{R}_{i}\left(\mathbf{U}_{\mathbf{i}}{ }^{2}\right)
\end{gathered}
$$

with $\mathbf{R}_{i}$ given by (13).

The time step $\Delta t$ is computed as follows

$$
\Delta t=K \min _{i} \frac{h_{i}}{S_{i} \cdot V_{i}}
$$

where $h_{i}$ is the radius of the inscribed sphere of each cell $i$ and $V_{i}$ its corresponding volume, $K \leq 1 / 3$ is the CFL number for unsplit finite-volume schemes [48] , and $S_{i}$ is the maximum propagation speed in each cell $i$ given by

$$
S_{i}=s p_{x} \cdot n_{x}+s p_{y} \cdot n_{y}+s p_{z} \cdot n_{z}
$$

where

$$
s p_{x}=|u+a|, \quad, s p_{y}=|v+a|, \quad s p_{x}=|w+a|,
$$

with $\mathbf{n}=\left(n_{x}, n_{y}, n_{z}\right)$ being the outward unit normal vector and $a$ is the speed of sound.

\section{Results}

This section presents the results obtained with the WENO schemes for a series of 2D and 3D test cases of a non-hydrostatic atmosphere.

\subsection{D Robert Smooth Bubble}

After its introduction [40], the Robert smooth bubble problem is considered as a standard benchmark in NWP. The evolution of a warm bubble in a constant potential temperature environment is investigated, and since the bubble is warmer than the ambient air, it rises and due to shearing motion it forms a mushroom shaped structure as shown in Figure 3. The twodimensional, non-hydrostatic compressible Euler equations (1) are numerically solved in the computational domain $1000 m \times 1500 m$ with $t \in[0,800] s$ with no flux boundary conditions. The initial condition [40] corresponds to a warm bubble centered at $(500,260) m$, in hydrostatic balance, and a perturbation of the potential temperature to trigger the movement of the warm bubble as follows: 


$$
\theta^{\prime}=\left\{\begin{array}{cl}
0 & \text { for } r<r_{c} \\
0.25\left(1+\cos \left(\frac{\pi r}{r_{c}}\right)\right) & \text { for } r \geq r_{c}
\end{array},\right.
$$

where $r$ is the distance from the centre of the bubble

$$
r=\sqrt{\left(x-x_{c}\right)^{2}+\left(z-z_{c}\right)^{2}}
$$

where $\pi$ is the trigonometric constant; $r_{c}=250 \mathrm{~m}$ is the radius of the bubble; and the potential temperature $\bar{\vartheta}$ is constant at $300 \mathrm{~K}$. The unstructured meshes used consist of arbitrary triangular elements as shown in Figure 2 and have resolutions of $25 \mathrm{~m}$ and $6 \mathrm{~m}$. The WENO3 and WENO5 schemes using characteristics based reconstructions are used, with a CFL number of 0.9.

From the obtained computational results as shown in Figure 3 and Figure 4 it can be noticed that long-wave oscillations of the interface occur forming smaller vortices, the spatial symmetry of the solution is completely lost and that higher-order WENO schemes resolve more of these small scale vortices.

Regarding the long-wave oscillations of the interface it can be attributed to the the shear flow interaction between the interface and the surrounding unperturbed air, which in turn give rise to the Kelvin-Helmholtz instabilities that lead to turbulent structures. There have been numerous extensive numerical experiments [20, 33, 40, 60] to investigate this problem and to date the exact solution remains unknown.

The spatial symmetry of the solution is lost due to the fact that the mesh is not symmetric, the initial profile is approximated by a Gaussian quadrature rule of the same order as the polynomial order used for the reconstruction, and finally due to the multidimensional reconstruction of the present algorithms. The fact that each time the mesh is refined, or a higher-order scheme (due to the Gaussian quadrature) is used the initial perturbations change slightly. In return these perturbations according to [20] grow exponentially in time, hence the perturbations have larger spatial scales that the initial perturbations.

As the grid resolution is refined or as the spatial order of the WENO schemes is increased the numerical dissipation is reduced, hence more structures are resolved. The question is whether or not these structures are just artificial or are part of the correct solution is an issue that requires further investigation, particularly in the absence of experiments. The WENO schemes for this discontinuous problem remain stable as witnessed by [38]. However, for other approaches that do not possess discontinuity capturing capabilities a form of artificial viscosity would be required both for stabilising and for obtaining a converged solution [33]. At the same time it can be realised that some of these perturbations may be part of the correct solution [33, 60], hence controlling the amount of artificial viscosity can be quite challenging.

\subsection{D Robert Smooth Bubble}

The three dimensional, non-hydrostatic compressible Euler equations (1) are numerically solved in the computational domain $1000 \mathrm{~m} \times 1000 \mathrm{~m} \times 1500 \mathrm{~m}$ and $t \in[0,450] \mathrm{s}$ in conjunction with NFBC. The initial condition [40] corresponds to a warm bubble centered at 


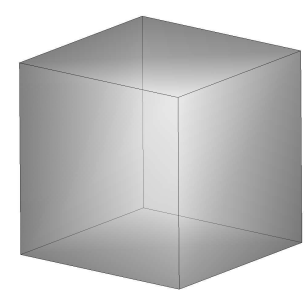

(a) Hexahedral

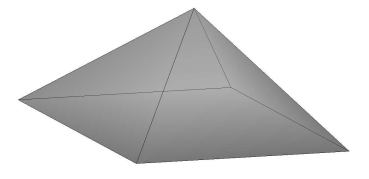

(b) Pyramid

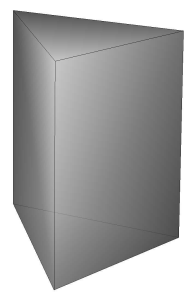

(c) Prism

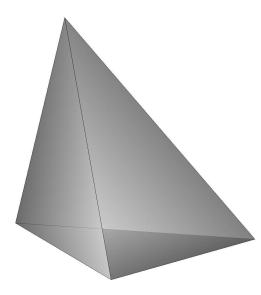

(d) Tetrahedral

Figure 1: 3D Element shapes

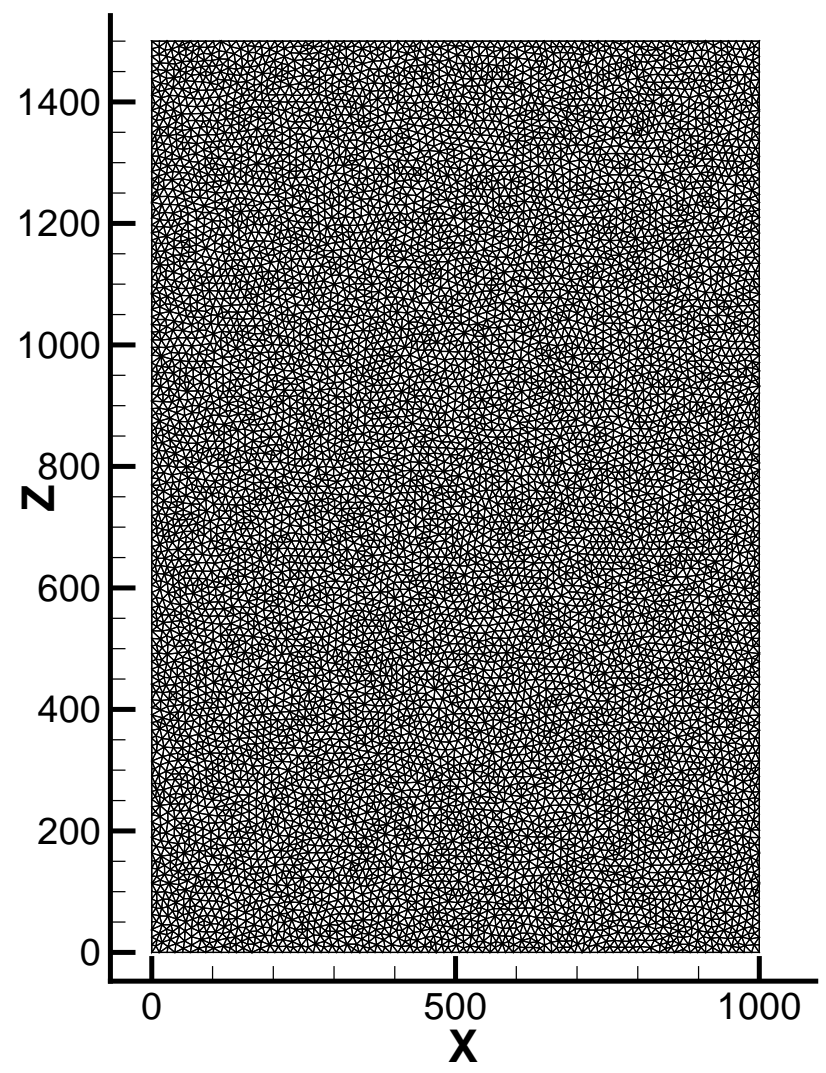

Figure 2: Unstructured mesh used for the rising thermal bubble case. 


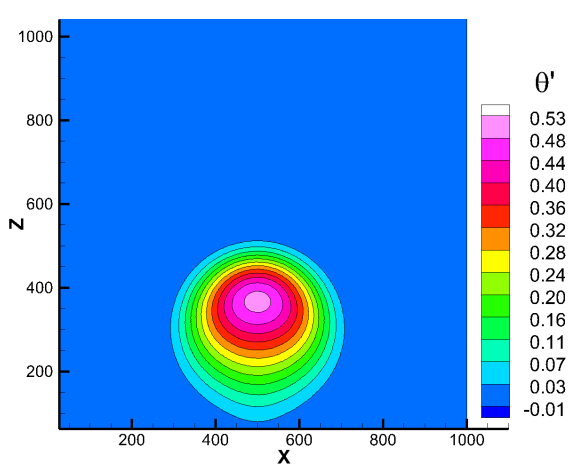

(a) $t=172 \mathrm{~s}$

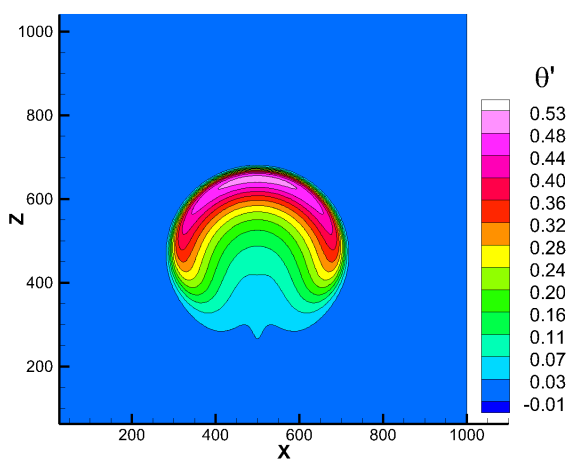

(c) $t=400 \mathrm{~s}$

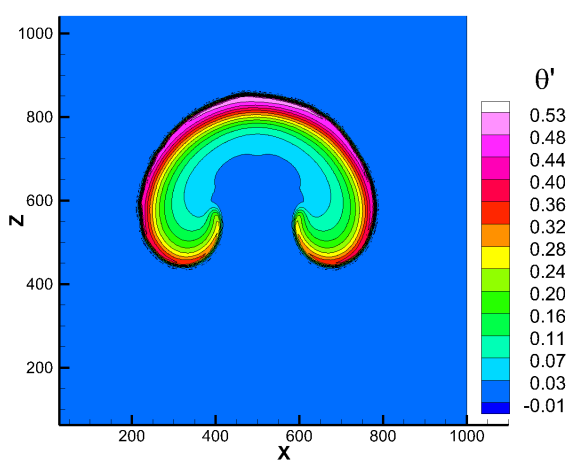

(e) $t=600 \mathrm{~s}$

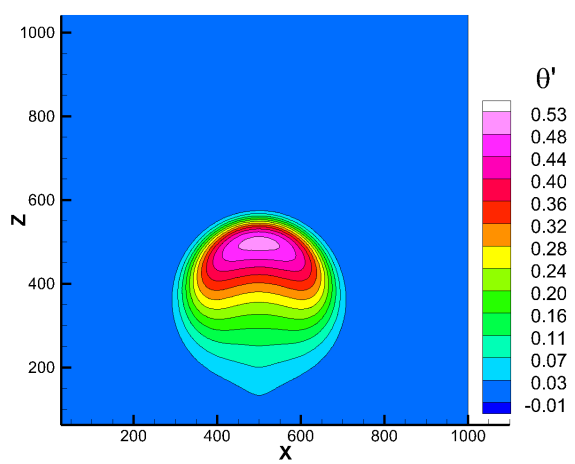

(b) $t=271 \mathrm{~s}$

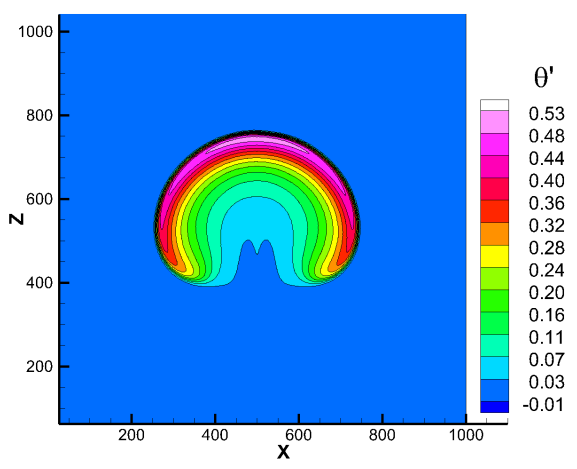

(d) $t=480 \mathrm{~s}$

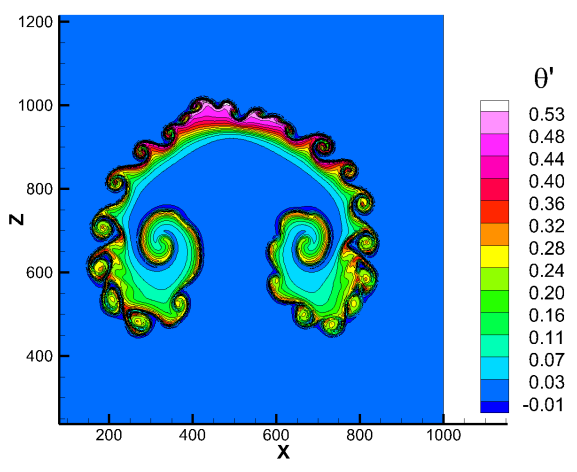

(f) $t=800 \mathrm{~s}$

Figure 3: Potential temperature perturbation $\theta^{\prime}(K)$ at various instants for the 2D Robert smooth bubble test on an unstructured mesh of $6 \mathrm{~m}$ resolution using a WENO5 scheme. 


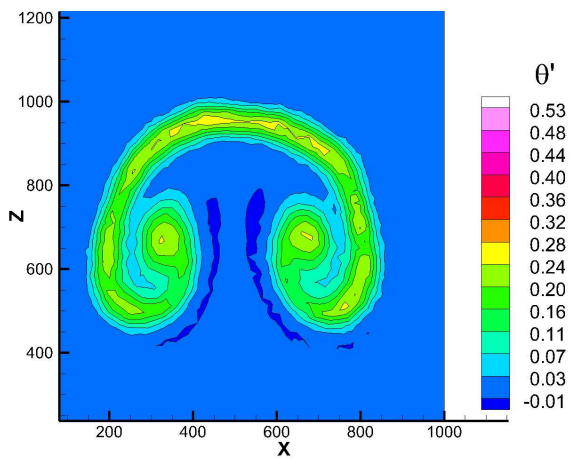

(a) $25 \mathrm{~m} \mathrm{WENO} 3$

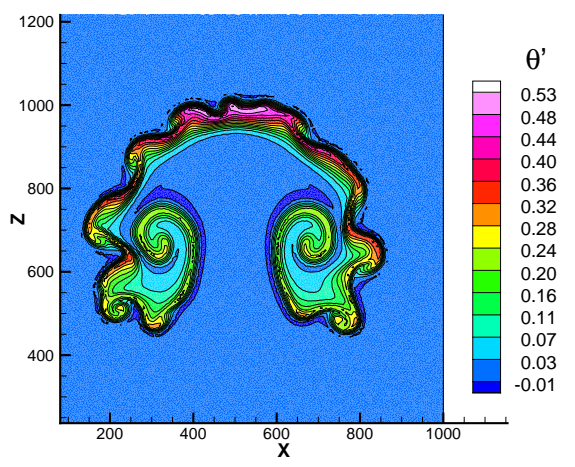

(c) $6 \mathrm{~m}$ WENO3

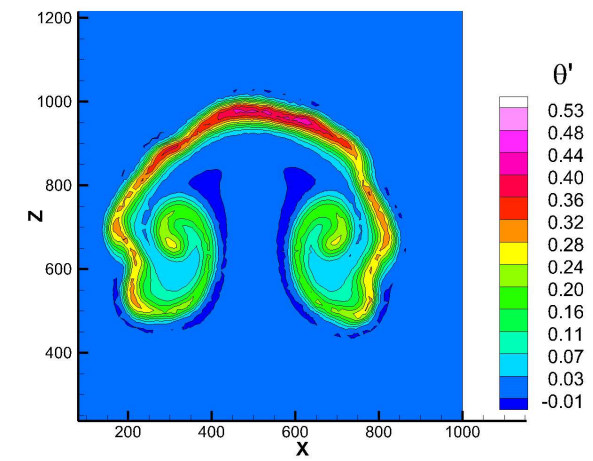

(b) $25 \mathrm{~m}$ WENO5

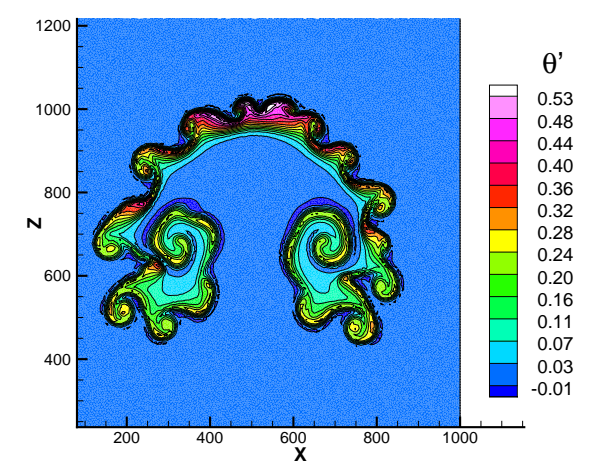

(d) $6 \mathrm{~m}$ WENO5

Figure 4: Potential temperature perturbation $\theta^{\prime}(K)$ at $t=800 \mathrm{sec} 2 \mathrm{D}$ Robert smooth bubble test at different grid resolutions with WENO3 and WENO5 schemes. 


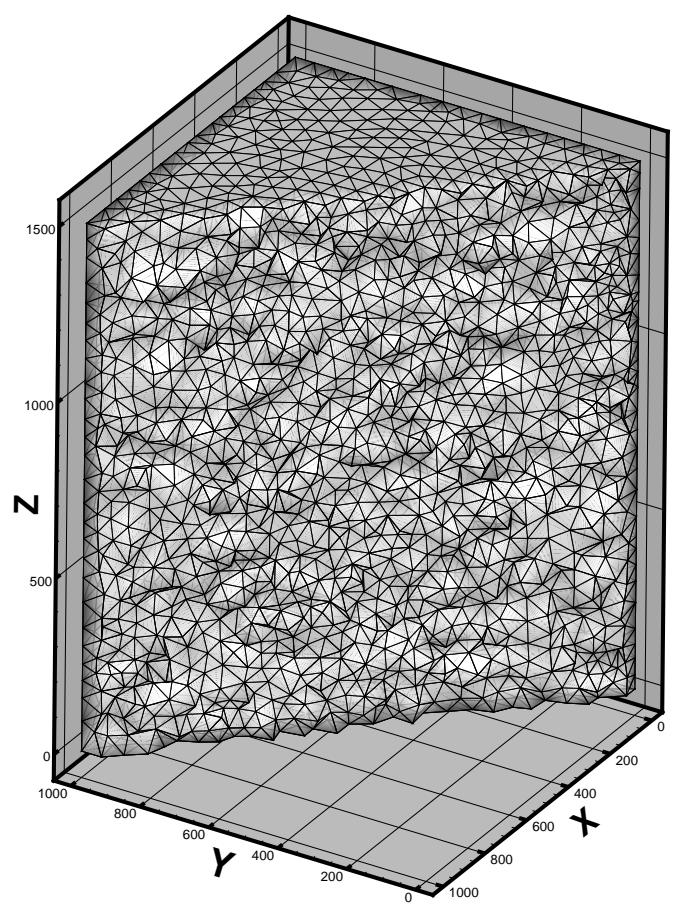

Figure 5: Cutaway of Unstructured mesh used for the 3D Robert smooth bubble test.

$(500,500,260) m$, in hydrostatic balance, and a perturbation of the potential temperature to trigger the movement of the warm bubble as follows:

$$
\theta^{\prime}=\left\{\begin{array}{cc}
0 & \text { for } r<r_{c} \\
1.25\left(1+\cos \left(\frac{\pi r}{r_{c}}\right)\right) & \text { for } r \geq r_{c}
\end{array}\right.
$$

where $r$ is the distance from the centre of the bubble

$$
r=\sqrt{\left(x-x_{c}\right)^{2}+\left(y-y_{c}\right)^{2}+\left(z-z_{c}\right)^{2}}
$$

$\pi$ is the trigonometric constant; $r_{c}=250$ is the radius of the bubble; and the potential temperature $\bar{\vartheta}$ is constant at $300 K$. Three unstructured meshes comprising of 19, 175, 64, 000 , and 210, 212 elements have been employed using tetrahedral elements Figure 5.

The main objective of this $3 \mathrm{D}$ test problem is to investigate if the performance of the WENO schemes at extremely low spatial resolution $30 \sim 100 \mathrm{~m}$, which is more realistic in terms of under resolved grid-arrangements used in NWP. The WENO3 and WENO5 schemes using characteristics based reconstructions are used with a CFL number of 0.9.

From the obtained computational results Figure 6, it can be noticed that the solution does not exhibit the same instabilities of the 2D test case, although the spatial symmetry of the solution is lost and that the higher-order WENO schemes provide sharper profile solutions. The non-existence of Kelvin-Helmholtz instabilities was expected due to the coarse grid resolution. 


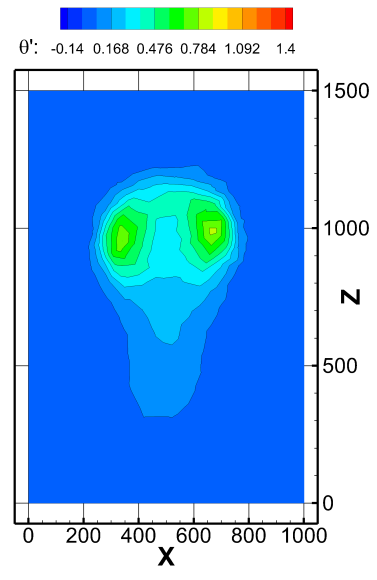

(a) $90 \mathrm{~m}$ WENO3

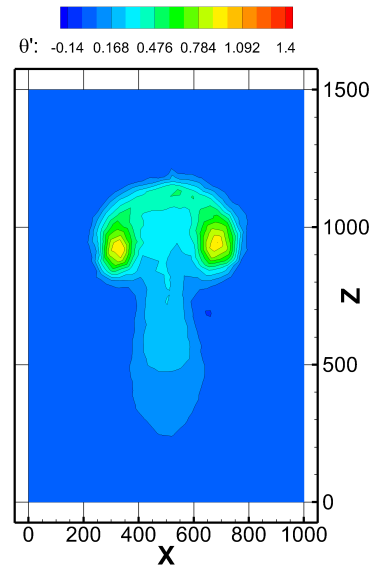

(d) $90 \mathrm{~m}$ WENO5

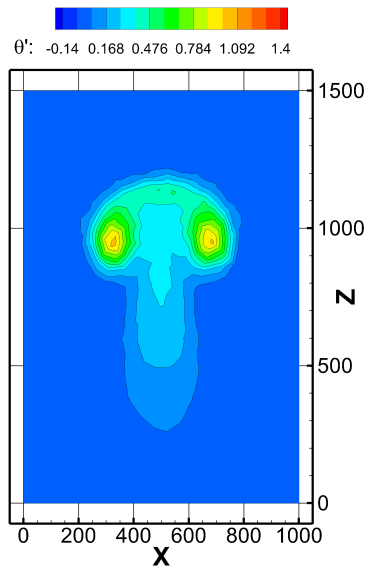

(b) $60 \mathrm{~m}$ WENO3

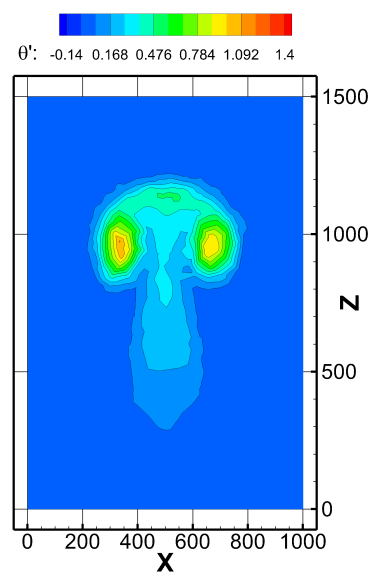

(e) $60 \mathrm{~m}$ WENO5

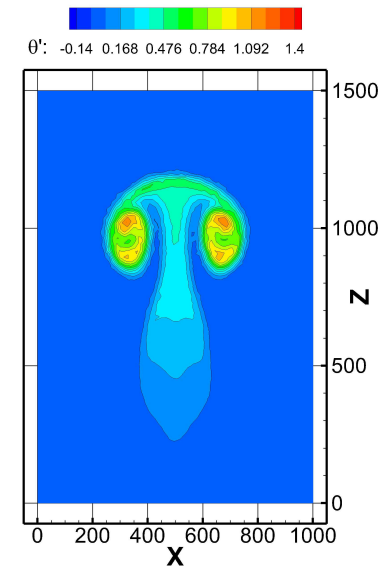

(c) $30 \mathrm{~m}$ WENO3

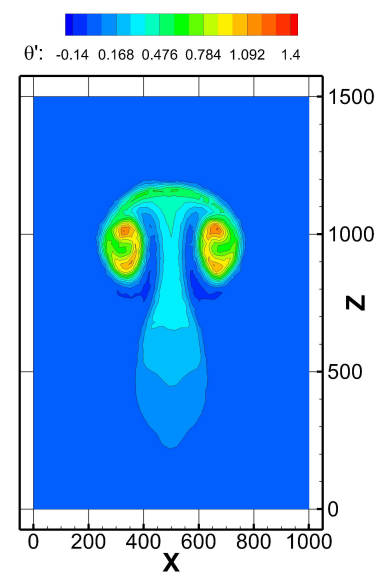

(f) $30 \mathrm{~m}$ WENO5

Figure 6: Potential temperature perturbation $\theta^{\prime}(K)$ at $t=300 \mathrm{sec}$ and $y=500 \mathrm{~m}$ for the 3D Robert smooth bubble test.

Despite the coarse resolution, the solution breaks up its symmnetry. The asymmetry of solution is more pronounced at coarser grid resolutions. This behaviour is attributed to the multidimensional character of the numerical reconstruction and is a well known property of high-order upwind schemes exhibited particularly in flows associated with vortical structures and separation. The Potential temperature perturbation $\theta^{\prime}(K)$ and vertical velocity $\mathbf{W}^{\prime}(\mathrm{m} / \mathrm{s})$ profiles (Figure 7) seem to trend towards convergence; although the solution is not expected to converge without any explicit viscosity due to the inviscid nature of the problem.

\subsection{Parallel Performance}

The requirements for atmospheric modelling on a regional or global scale have significantly increased in the last decade. Parallelisation of existing computational methods and software is an active area of research. Previous studies [50] showed that the ratio of computational time 


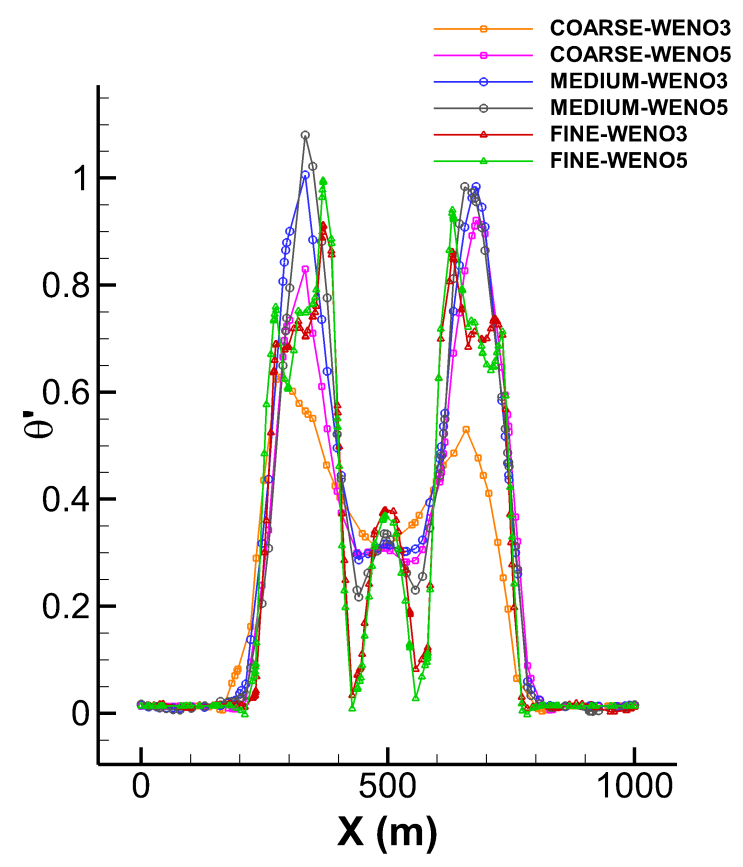

(a) $\theta^{\prime}(K)$

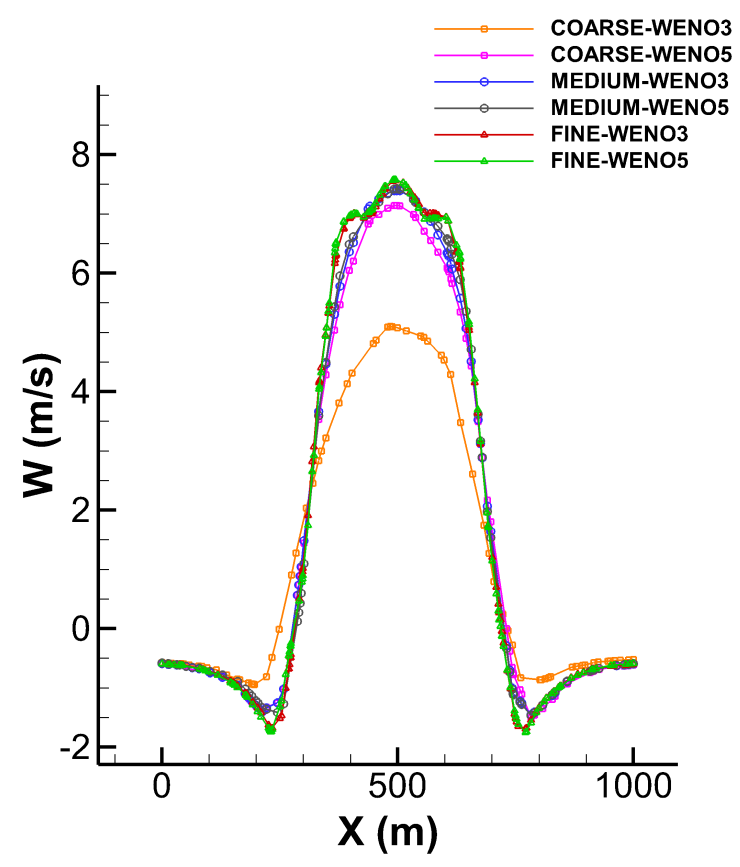

(b) $\mathbf{W}(\mathrm{m} / \mathrm{s})$

Figure 7: Potential temperature perturbation $\theta^{\prime}(K)$ and vertical velocity $\mathbf{W}(\mathrm{m} / \mathrm{s})$ at $t=300 \mathrm{sec}$ $y=500 m$ and $z=964.5 m$ for the Robert smooth bubble test. 


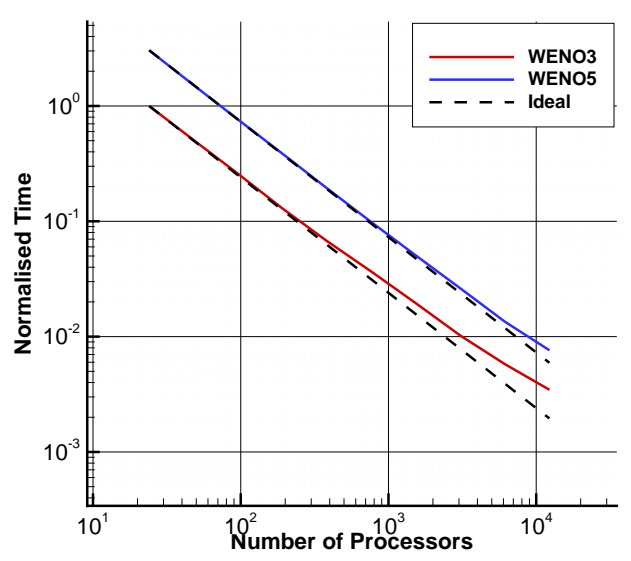

(a) Scalability

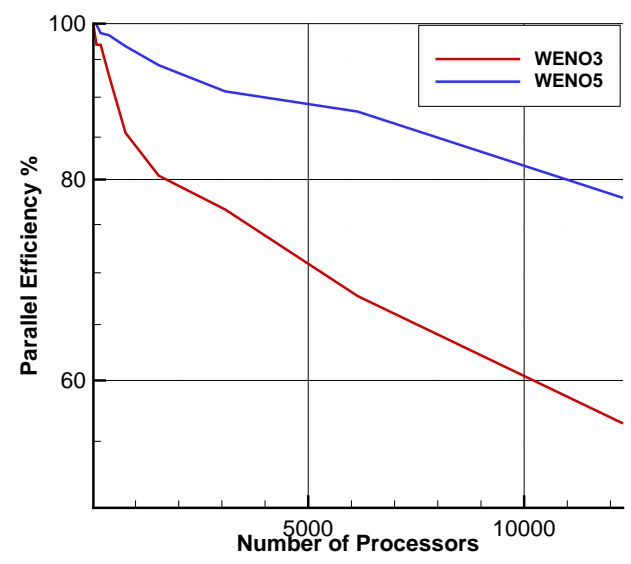

(b) Efficiency

Figure 8: Scalability and efficiency of WENO3 and WENO5 methods

over communication time is proportional to the order of accuracy of the WENO scheme. For WENO schemes with accuracy greater than third, the computational time is one order of magnitude greater than the communication time required for each iteration. One of the advantages of explicit methods used here is that they can be easily parallelised using domain decomposition. The software package used for partitioning uniform and hybrid unstructured meshes is the METIS software package [25]. The present numerical methods are employed in an unstructured flow solver (UCNS3D) capable of handling hybrid meshes in three-dimensions.

The Robert smooth bubble using the finest resolution, unstructured mesh with 210, 212 elements, was utilised as a test problem to assess the scalability of the developed numerical schemes. Although this grid resolution for this sample test case is relatively coarse when compared to the grid resolutions employed for climate and numerical weather prediction, our primary aim for is to demonstrate how much dependent the parallel performance is on numerical schemes implemented in the same software framework. The performance tests were conducted at the ARCHER UK National Supercomputing Service at EPCC. The results were obtained using the latest Intel FORTRAN compiler and Intel MPI library.

The time required for each time-step for the WENO3 and WENO5 schemes with respect to the number of processors is illustrated in Figure 8. The time is normalised with the time required for the WENO3 scheme.

The results confirm that high order methods scale better than lower order methods due to the fact that the ratio of computational time over communication time is greater in the lower order methods. Using 6144 processors, the parallel efficiency for the different schemes is: WENO3 (67\%) and WENO5 (87\%). It is pointed out that for the WENO5 scheme $92 \%$ of the computational time is spent in the reconstruction process. The exchange of the updated values of the stencil elements takes less than $3 \%$ of the total communication time since the largest communication requirement is the exchange of the reconstructed values for each quadrature point of halo-cells. The WENO high-order schemes have a greater computational cost, but 
are expected to be more accurate when problems with large time scales are involved such as numerical climate modelling. For numerical climate modelling where projections of the climate for centuries are studied, WENO schemes are expected to have the strength of sufficiently small dissipation errors that will not contaminate the projected solution as a lower-order numerical scheme.

When using 6,144 processors, each processor holds approximately 35 elements and on 12, 288 processors, each processor has less than 18 elements. The latter seems extremely low, however, it may become common practice in the future when exaflop systems will become available. These results also illustrate that improving the scalability and efficiency of numerical weather prediction models in order to take advantage of massive parallel systems, does not only require the optimisation of the computational software (software engineering), but also requires re-designing numerical methods in order to make most of the parallel performance.

\section{Conclusions}

We presented the implementation of high-order finite-volume schemes for atmospheric dynamics on unstructured meshes. Combining the features of unstructured meshes to accurately represent complex topologies that can be extended to adapt the resolution (dynamically or statically) in certain flow regions and using high-order numerical methods that utilise only local information, results in highly-efficient and highly-accurate numerical frameworks for numerical weather and climate modelling.

The present study discussed the implementation of WENO numerical schemes in this context and assessed their performance in terms of accuracy, robustness, efficiency and scalability. The results demonstrate the aforementioned properties of the schemes in the two-dimensional and three-dimensional rising bubble test cases, and the associated challenges of multidimensional discretisations. The WENO reconstruction can be utilised as a building-block in a dynamical core that is not limited by the type of meshes, or the type of the governing equations. Future work will concern application of the schemes in regional and global atmospheric simulations.

\section{Acknowledgments}

The authors acknowledge the computing time on the UK national high-performance computing service ARCHER that was provided through the UK turbulence consortium in the framework of the EPSRC grant EP/G069581/1. Panagiotis Tsoutsanis acknowledges PRACE for awarding him access to resource SUPERMUC and HazelHen based in Germany at Munich and Stuttgart respectively and the support of Thomas Ponweiser from RISC Software GmbH, Austria to the technical work is gratefully acknowledged.

\section{References}

[1] R. Abgrall. On essentially non-oscillatory schemes on unstructured meshes: analysis and implementation. Journal of Computational Physics, 114(1):45-58, 1994.

[2] A. F. Antoniadis, D. Drikakis, I. Kokkinakis, P. Tsoutsanis, and Z. Rana. High-order methods for hypersonic shock wave turbulent boundary layer interaction flow. In AIAA, 
editor, 20th AIAA International Space Planes and Hypersonic Systems and Technologies Conference, number AIAA 2015-3524, Glasgow, Scotland, July 2015.

[3] A. F. Antoniadis, P. Tsoutsanis, and D. Drikakis. Numerical accuracy in rans computations of high-lift multi-element airfoil and aircraft configurations. In AIAA, editor, 53rd AIAA Aerospace Sciences Meeting, number AIAA 2015-0317, Kissimmee, Florida, January 2015.

[4] S. Brdar, M. Baldauf, A. Dedner, and R. Klofkorn. Comparison of dynamical cores for nwp models: comparison of cosmo and dune. Theoretical and Computational Fluid Dynamics, 27:453-472, 2013.

[5] C. Breviglieri, A.F.S. Maximiliano, E. Basso, and J.L.F. Azevedo. Improved high-order spectral finite volume method implementation for aerodynamic flows. 2009.

[6] B. Cockburn and C.-W. Shu. Runge-kutta discontinuous galerkin methods for convectiondominated problems. Journal of Scientific Computing, 16(3):173-261, 2001.

[7] J.M. Dennis, R.D. Nair, H.M. Tufo, M. Levy, and T. Voran. Development of a scalable global discontinuous galerkin atmospheric model. Int. J. Comp. Sci. Eng.

[8] D. Drikakis, A. F. Antoniadis, P. Tsoutsanis, I. Kokkinakis, and Z. Rana. Azure: An advanced cfd software suite based on high-resolution and high-order methods. In AIAA, editor, 53rd AIAA Aerospace Sciences Meeting, number AIAA 2015-0813, Kissimmee, Florida, January 2015.

[9] M. Dumbser and M. Kaser. Arbitrary high order non-oscillatory finite volume schemes on unstructured meshes for linear hyperbolic systems. Journal of Computational Physics, 221(2):693-723, 2007.

[10] M. Dumbser, M. Kaser, V.A. Titarev, and E.F. Toro. Quadrature-free non-oscillatory finite volume schemes on unstructured meshes for nonlinear hyperbolic systems. Journal of Computational Physics, 226(1):204-243, 2007.

[11] M. Dumbser, D.S. Balsara, E.F. Toro, and C.-D. Munz. A unified framework for the construction of one-step finite volume and discontinuous galerkin schemes on unstructured meshes. Journal of Computational Physics, 227(18):8209-8253, 2008.

[12] M. Dumbser, A. Hidalgo, and O. Zanotti. High order space-time adaptive ader-weno finite volume schemes for non-conservative hyperbolic systems. Computer Methods in Applied Mechanics and Engineering, 268:359-387, 2014.

[13] O. Friedrich. Weighted essentially non-oscillatory schemes for the interpolation of mean values on unstructured grids. Journal of Computational Physics, 144(1):194-212, 1998.

[14] F. X. Giraldo, J. F. Kelly, and E. M. Constantinescu. Implicit-explicit formulations of a three-dimensional nonhydrostatic unified model of the atmosphere (numa). SIAM Journal of Scientific Computing, 35:B1162-B1194, 2013. 
[15] F.X. Giraldo and M. Restelli. A study of spectral element and discontinuous galerkin methods for the navier-stokes equations in nonhydrostatic mesoscale atmospheric modeling: Equation sets and test cases. Journal of Computational Physics, 227(8):3849-3877, 2008.

[16] F.X. Giraldo, M. Restelli, and M. Louter. Semi-implicit formulations of the navier-stokes equations: Application to nonhydrostatic atmospheric modeling. SIAM Journal on Scientific Computing, 32(6):3394-3425, 2010.

[17] Giraldo, F. Non-hydrostatic Unified Model of the Atmosphere (NUMA), 2015. URL http://faculty.nps.edu/fxgirald/projects/NUMA/Introduction_to_NUMA.html.

[18] C. Ollivier Gooch and M. Van Altena. A high-order-accurate unstructured mesh finitevolume scheme for the advection-diffusion equation. Journal of Computational Physics, 181(2):729-752, 2002.

[19] S. Gottlieb. On high order strong stability preserving runge-kutta and multi step discretizations. Journal of Scientific Computing, 25(112):105-128, 2005.

[20] W.W. Grabowski and T.L. Clark. Cloud-environment interface instablility: rising thermal calculations in two spatial dimensions. Journal of Atmospheric Sciences, 48(1):527-546, 1991.

[21] A. Haselbacher. A weno reconstruction algorithm for unstructured grids based on explicit stencil construction. pages 3369-3378, 2005.

[22] G. Hu, R. Li, and T. Tang. A robust weno type finite volume solver for steady euler equations on unstructured grids. Communications in Computational Physics, 9(3):627648, 2011.

[23] G. S Jiang and C. W Shu. Efficient implementation of weighted eno schemes. Journal of Computational Physics, 126(1):202-228, 1996.

[24] D. Kalise and I. Lie. Modeling and numerical approximation of a $2.5 \mathrm{~d}$ set of equations for mesoscale atmospheric processes. Journal of Computational Physics, 231:7274-7298, 2012.

[25] G. Karypis and V. Kumar. Multilevel k-way partitioning scheme for irregular graphs. $J$. Parallel Distrib. Comput., 48:96-129, 1998.

[26] J.F. Kelly, F.X. Giraldo, and G. Jost. Scalability of semi-implicit time integrators for nonhydrostatic galerkin-based atmospheric models on large scale clusters. pages 561$565,2011$.

[27] B. Van Leer. Towards the ultimate conservative difference scheme. ii. monotonicity and conservation combined in a second-order scheme. J. Comput. Phys., 14:361-370, 1974. 
[28] B. Van Leer. Towards the ultimate conservative difference scheme. v. a second-order sequel to godunov's method. J. Comput. Phys., 32(1):101-136, 1979.

[29] W.A. Li and Y.X. Ren. Quadrature-free non-oscillation finite volume scheme for solving navier-stokes equations on unstructured grids. volume 1376, pages 639-641, 2011.

[30] S. Marras, M. Moragues, M. Vzquez, O. Jorba, and G. Houzeaux. A variational multiscale stabilized finite element method for the solution of the euler equations of nonhydrostatic stratified flows. Journal of Computational Physics, 236(1):380-407, 2013.

[31] Max Planck Institute for Metereology and Deutscher Wetterdienst. ICOsahedral Non-hydrostatic General Circulation Model, 2015. URL http://www.mpimet.mpg.de/en/science/models/icon.html.

[32] A. Muller, J. Behrens, F. X. Giraldo, and V. Wirth. Comparison between adaptive and uniform discontinuous galerkin simulations in dry $2 \mathrm{~d}$ bubble experiments. Journal of Computational Physics, 235:371-393, 2013.

[33] A. Muller, J. Behrens, F.X. Giraldo, and V. Wirth. Comparison between adaptive and uniform discontinuous galerkin simulations in dry $2 \mathrm{~d}$ bubble experiments. Journal of Computational Physics, 235:371-393, 2013.

[34] National Center for Atmospheric Research. High-Order Methods Modeling Environment, 2015. URL http://www.homme.ucar.edu/

[35] National Oceanic Atmospheric Administration. Am3, 2015. URL http://www.gfdl.noaa.gov/am3

[36] National Oceanic Atmospheric Administration. Nonhydrostatic Icosahedral Model NIM, 2015. URL http://www.esrl.noaa.gov.

[37] X. Nogueira, L. Cueto-Felgueroso, I. Colominas, F. Navarrina, and M. Casteleiro. A new shock-capturing technique based on moving least squares for higher-order numerical schemes on unstructured grids. Computer Methods in Applied Mechanics and Engineering, 199(37-40):2544-2558, 2010.

[38] M.R. Norman, R.D. Nair, and F.H.M. Semazzi. A low communication and large time step explicit finite-volume solver for non-hydrostatic atmospheric dynamics. Journal of Computational Physics, 230(4):1567-1584, 2011.

[39] C.F. Ollivier-Gooch. Quasi-eno schemes for unstructured meshes based on unlimited datadependent least-squares reconstruction. Journal of Computational Physics, 133(1):6-17, 1997.

[40] A. Robert. Bubble convection experiments with a semi-implicit formulation of the euler equations. Journal of the Atmospheric Sciences, 50(13):1865-1873, 1993. 
[41] M. Satoh, T. Matsuno, H. Tomita, H. Miura, T. Nasuno, and S. Iga. Nonhydrostatic icosahedral atmospheric model (nicam) for global cloud resolving simulations. Journal of Computational Physics, 227(7):3486-3514, 2008.

[42] J. Shi, C. Hu, and C.-W. Shu. A technique of treating negative weights in weno schemes. Journal of Computational Physics, 175(1):108-127, 2002.

[43] C.-W. Shu and S. Osher. Efficient implementation of essentially non-oscillatory shockcapturing schemes. Journal of Computational Physics, 77(2):439-471, 1988.

[44] P. K. Smolarkiewicz, Szmelter J., and A. A. Wyszogrodzki. An unstructured-mesh atmospheric model for nonhydrostatic dynamics. Journal of Computational Physics, 254: 184-199, 2013.

[45] P.K. Smolarkiewicz. Multidimensional positive definite advection transport algorithm: An overview. International Journal for Numerical Methods in Fluids, 50(10):1123-1144, 2006.

[46] D. Stanescu and W.G. Habashi. Essentially nonoscillatory euler solutions on unstructured meshes using extrapolation. AIAA Journal, 36(8):1413-1416, 1998.

[47] V.A. Titarev, P. Tsoutsanis, and D. Drikakis. Weno schemes for mixed-element unstructured meshes. Communications in Computational Physics, 8(3):585-609, 2010.

[48] Eleuterio F. Toro. Riemann Solvers and Numerical Methods for Fluid Dynamics - A Practical Introduction. Berlin: Springer-Verlag, 1997.

[49] P. Tsoutsanis, V.A. Titarev, and D. Drikakis. Weno schemes on arbitrary mixed-element unstructured meshes in three space dimensions. Journal of Computational Physics, 230 (4):1585-1601, 2011.

[50] P. Tsoutsanis, A.F. Antoniadis, and D. Drikakis. Weno schemes on arbitrary unstructured meshes for laminar, transitional and turbulent flows. Journal of Computational Physics, 256:254-276, 2014.

[51] UK MET Office and ECMWF. Even Newer Dynamics for General atmospheric modelling of the environment (ENDGame), $2015 . \quad$ URL http://www.metoffice.gov.uk/research/areas/dynamics/endgame.

[52] A. Uranga, P.-O. Persson, M. Drela, and J. Peraire. Implicit large eddy simulation of transition to turbulence at low reynolds numbers using a discontinuous galerkin method. International Journal for Numerical Methods in Engineering, 87(1-5):232-261, 2011.

[53] Z.J. Wang. Spectral (finite) volume method for conservation laws on unstructured grids. basic formulation. Journal of Computational Physics, 178(1):210-251, 2002. 
[54] Z.J. Wang and Y. Liu. Spectral (finite) volume method for conservation laws on unstructured grids. ii. extension to two-dimensional scalar equation. Journal of Computational Physics, 179(2):665-697, 2002.

[55] Z.J. Wang, L. Zhang, and Y. Liu. Spectral (finite) volume method for conservation laws on unstructured grids iv: Extension to two-dimensional systems. Journal of Computational Physics, 194(2):716-741, 2004.

[56] W.R. Wolf and J.L.F. Azevedo. High-order eno and weno schemes for unstructured grids. International Journal for Numerical Methods in Fluids, 55(10):917-943, 2007.

[57] Z. Xu, Y. Liu, and C.-W. Shu. Hierarchical reconstruction for discontinuous galerkin methods on unstructured grids with a weno-type linear reconstruction and partial neighboring cells. Journal of Computational Physics, 228(6):2194-2212, 2009.

[58] Z. Xu, Y. Liu, and C.-W. Shu. Hierarchical reconstruction for spectral volume method on unstructured grids. Journal of Computational Physics, 228(16):5787-5802, 2009.

[59] C. Yang and X. C. Cai. A scalable fully implicit compressible euler solver for mesoscale nonhydrostatic simulation of atmospheric flow. SIAM Journal of Scientific Computing, 36: S23-S47, 2014.

[60] L. Yelash, A. Muller, M. Lukacova-Medvid'ova, F.X. Giraldo, and V. Wirth. Adaptive discontinuous evolution galerkin method for dry atmospheric flow. Journal of Computational Physics, 268:106-133, 2014.

[61] G. Zangl, D. Reinert, P. Ripodas, and M. Baldauf. The icon (icosahedral non-hydrostatic) modelling framework of dwd and mpi-m: Description of the non-hydrostatic dynamical core. Quarterly Journal of the Royal Meteorological Society, 2014.

[62] Y. T. Zhang and C. W. Shu. Third order weno scheme on three dimensional tetrahedral meshes. Communications in Computational Physics, 5(2-4):836-848, 2008.

[63] Y. Zhou and Z.J. Wang. Implicit large eddy simulation of transitional flow over a sd7003 wing using high-order spectral difference method. 2010.

[64] J. Zhu, J. Qiu, C.-W. Shu, and M. Dumbser. Runge-kutta discontinuous galerkin method using weno limiters ii: Unstructured meshes. Journal of Computational Physics, 227(9): 4330-4353, 2008. 


\section{Addressing the challenges of} implementation of high-order finite volume schemes for atmospheric dynamics of unstructured meshes

\section{Tsoutsanis, Panagiotis}

European Congress on Computational Methods in Applied Sciences and Engineering

Tsoutsanis, P., Drikakis, D. (2016) Addressing the challenges of implementation of high-order finite-volume schemes for atmospheric dynamics of unstructured meshes, Proceedings of the ECCOMAS Congress 2016 VII European Congress on Computational Methods in Applied Sciences and Engineering, 5-10 June 2016, Crete, Greece.

http://www.eccomas2016.org/proceedings/pdf/8406.pdf

Downloaded from Cranfield Library Services E-Repository 